\title{
Adenocarcinoma at previous gastrojejunostomy site
}

\author{
Saroj Kant Sahoo, Manash Ranjan Sahoo, Ved Bhaskar
}

\begin{abstract}
Introduction: There has been a growing interest in the development of gastric cancer following gastric surgery for benign disease. However, the risk is only observed after a latency of about 15 years and is increased in patients operated for gastric but not duodenal ulcers. The incidence of malignancy ranges from 2-6\% in gastric remnants and a variety of causative factors have been proposed such as alkaline duodenal gastric reflux and increased $\mathrm{N}$-nitroso compounds secondary to bacterial overgrowth. Case Report: We report an unusual case of a 75-year-old female who presented with recurrent vomiting. She had a gastrojejunostomy without gastric resection 25 years back. On investigation a gastric cancer was found at the anastomotic site in the efferent loop. Conclusion: Patients who have undergone surgery for ulcers should be screened using endoscopy at least once a year to detect the cancer in early stages as symptoms of cancer appear quite late and mortality is very high in advanced cases of stomach carcinoma. A complete margin negative (Ro) resection offers chances of cure in these patients.
\end{abstract}

Saroj Kant Sahoo ${ }^{1}$, Manash Ranjan Sahoo ${ }^{2}$, Ved Bhaskar ${ }^{3}$ Affiliations: ${ }^{1}$ Professor, Department of Surgery, S.C.B. Medical College, Cuttack, Odisha, India; ${ }^{2}$ Associate Professor, Department of Surgery, S.C.B. Medical College, Cuttack, Odisha, India; ${ }^{3}$ Post Graguate, Department of Surgery, S.C.B. Medical College, Cuttack, Odisha, India. Corresponding Author: Dr. Manash Ranjan Sahoo, Mailing Address: Associate professor, Department of Surgery, S.C.B Medical College, Cuttack, Odisha, India. Postal Code: 753007; Ph: +919937025779; Fax: 0671-2414034; Email: manash67@gmail.com

Received: 16 August 2012

Accepted: 17 October 2012

Published: 01 February 2013
Keywords: Gastrojejunostomy, Peptic ulcer, Gastric adenocarcinoma, Stump carcinoma

$* * * * * * * * *$

Sahoo SK, Sahoo MR, Bhaskar V. Adenocarcinoma at previous gastrojejunostomy site. International Journal of Case Reports and Images 2013;4(2):127-130.

$* * * * * * * * *$

doi:10.5348/ijcri-2013-02-277-CR-11

\section{INTRODUCTION}

There has been a growing interest in the development of gastric cancer following gastric surgery for benign disease. However, the risk is only observed after a latency of about 15 years and is increased in patients operated for gastric ulcers but not duodenal ulcers. The incidence of malignancy ranges from 2-6\% in gastric remnants and a variety of causative factors have been proposed including alkaline duodenal gastric reflux and increased N-nitroso compounds secondary to bacterial overgrowth [1].

We report an unusual case of gastric cancer occurring at the gastrojejunostomy anastomotic site, 25 years after gastrojejunostomy without gastric resection, in a 75-year-old female presenting with gastric outlet obstruction.

\section{CASE REPORT}

A 75-year-old female presented to us with complaints of recurrent vomiting, anorexia and loss of weight since two months. Vomiting was projectile in nature, non bilious, contained food particles, and usually occurred immediately after taking food. Gradually, patient developed aversion to food and was on liquid diet since last one month. She gave a history of 
gastrojejunostomy without gastrectomy for peptic ulcer disease which way done 25 years back. At presentation the patient was conscious and cooperative. Vitals signs were stable. She was thinly built with mild pallor present. On physical examination, adomen was scaphoid and umbilicus was centrally placed and inverted. A midline scar was seen extending from $5 \mathrm{~cm}$ below the xiphisternum to the umbilicus. Visible gastric peristalsis were seen. A firm, irregular, non-tender mass about $2 \times 1 \mathrm{cms}$ was felt in midline above the umbilicus which moved with respiration. Liver was not enlarged. Spleen was not palpable. No other organomegaly or free fluid was present. Per rectal examination revealed no abnormality. Routine laboratory investigations showed hemoglobin $8 \mathrm{~g} / \mathrm{dL}$, serum protein $5.0 \mathrm{~g} / \mathrm{dL}$ and serum albumin $2.8 \mathrm{~g} / \mathrm{dL}$. All other investigations were within normal limits. Ultrasonography of abdomen did not reveal any significant findings. Computed tomography (CT) scan of abdomen revealed irregular posterior gastric wall thickening with in-situ infiltration involving the gastrojejunostomy site. No evidence of any secondary spread to solid viscera or any enlarged node was seen. Upper gastrointestinal endoscopy showed gastrointestinal stoma with stomal ulcerated growth and pyloric stenosis. Endoscopic biopsy was taken which on histopathological examination, showed invasive adenocarcinoma of tubular type grade II, (moderately differentiated).

The patient was taken up for surgery after correction of anemia and hypoproteinemia by blood transfusion, fresh frozen plasma and albumin infusion. A distal subtotal gastrectomy and D1 lymph node dissection and resection of previously anastomosed jejunal stoma (Figure 1) with Roux-en-Y anastomosis was done. The growth was found after opening the specimen at the gastrojejunostomy stoma (Figure 2). Postoperative recovery was uneventful. She was started on liquid diet on postoperative day 4 and semisolid diet on day 7 . The patient was discharged on 12 postoperative day.

Histopathology report gave a diagnosis of gastric carcinoma stage II (T2b N1 Mx).

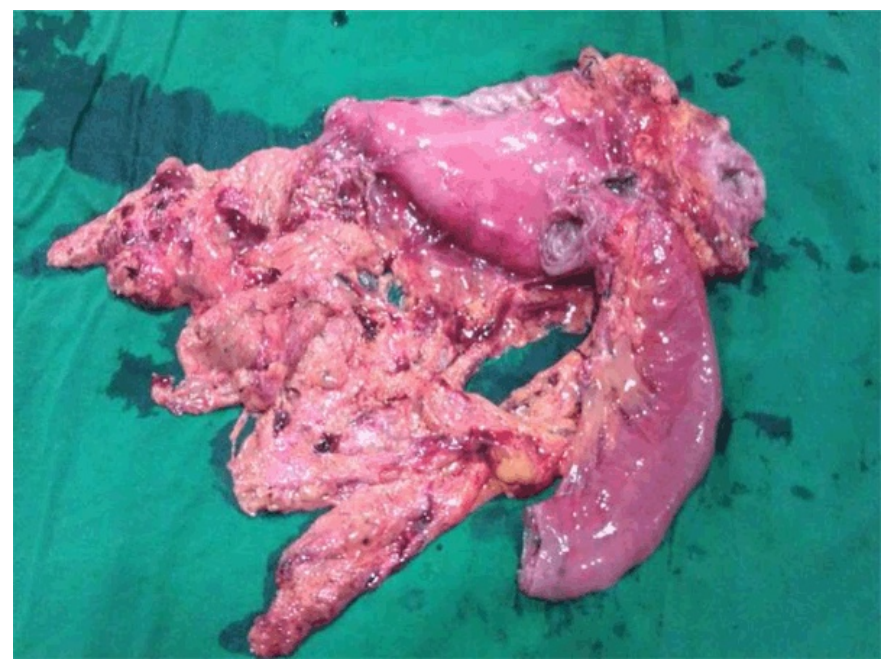

Figure 1: The subtotal gastrectomy specimen with $\mathrm{D}$, lymph node dissection and resection of anastomosed jejunal stoma.

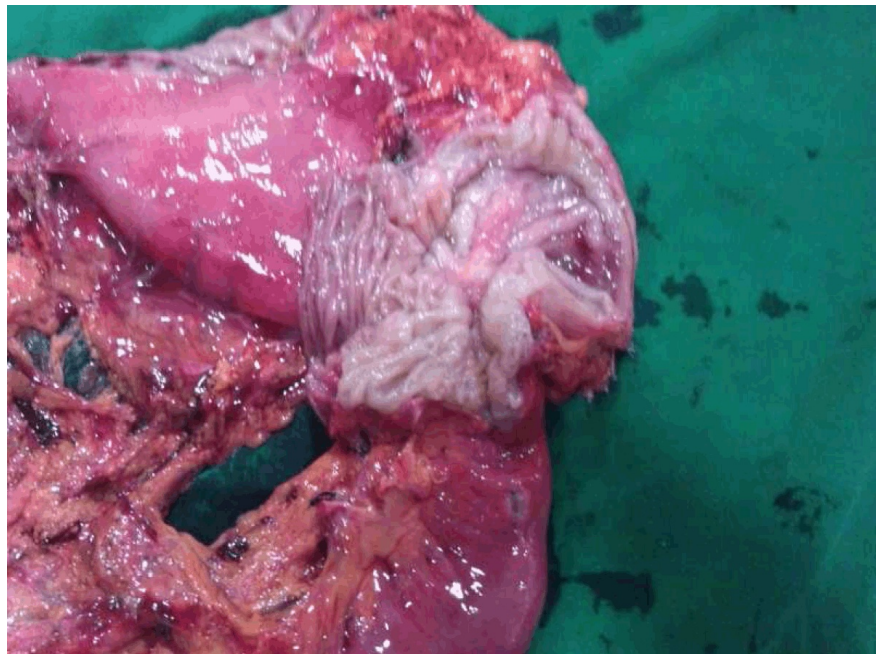

Figure 2: Gross appearance of malignancy seen at the gastrojejunostomy stoma.

\section{DISCUSSION}

It has been observed that patients who undergo gastrojejunostomy for peptic ulcer disease are at increased risk for gastric carcinoma [2]. Gastric cracinoma usually arise in an area of chronic gastritis, metaplasia and dysplasia which often occurs near the stoma. Most cases of stump carcinoma have been reported following Billroth II gastroenterostomy, but cases have also been reported following Billroth I gastroduodenostomy [1].

Gastric stump carcinoma is defined as an adenocarcinoma of the stomach occurring 10 years or more after gastrectomy for cancer or benign disease. It has been observed that patients with gastrectomy for cancer developed stump carcinoma in a significantly shorter time span than those with gastrectomy for benign diseases. The reason for these differences is that patients with gastric carcinoma already have carcinomarelated gastric mucosal changes at the time of primary surgery. Thus, gastric stump carcinoma after benign disease develops as a new lesion, while that after malignant disease may be a metachronous multiple lesion $[1,3]$.

Patients with Billroth II gastrectomy also develop stump carcinoma most frequently in the anastomotic area, regardless of the character of the original disease, whereas carcinoma for those with Billroth I gastrectomy is more frequently located in the non-stump area. The increased risk with the Billroth II procedure has been attributed to continuous bathing of the gastric stump anastomosis with bile acids, resulting in mucosal inflammation and regeneration [1]. The location of stump carcinoma close to the Billroth II stoma suggests involvement of duodenogastric reflux in gastric carcinogenesis [4].

There are differences in the tumor histology of gastric stump carcinomas between the early stage in which carcinomas are mostly intestinal type and the 
locally advanced stage in which the carcinomas are mostly diffuse type. This suggest that stump carcinoma may develop as intestinal type and later change to diffuse type during the evolution to advanced stage cancers $[1,4]$.

In 1972, Littler et al. reported that polypoid tumor developed in human gastric mucosa adjacent to Billroth II stoma. Based on the characteristic changes in hyperplastic foveolar epithelium and the multiple cystically dilated glands, they termed these tumors "gastritis cystica polyposa" (GCP) [3]. The etiology and pathogenesis of GCP are considered to be chronic inflammation; a consequence of the suture technique itself; or a consequence of duodenogastric reflux into the gastric remnant. In 1975, for the first time, Qizilbash reported the association of GCP with stump carcinoma [5]. In Japan, several reports have documented GCP associated with early gastric stump carcinoma after Billroth II procedure [6, 7 ]. Characteristically, the foci of adenocarcinoma are found in the superficial layers of GCP. The degree of intestinal metaplasia in the surrounding mucosa was low. These findings suggest a relationship between GCP and gastric type adenocarcinoma [3].

As far as role of H. Pylori and Estein Barr Virus in the pathogenesis of stomach carcinoma is concerned, two important differences were observed between gastric stump carcinomas and conventional primary stomach cancers. H. pylori infection, which is associated with the occurrence of cancer in the intact stomach, appears rare near the anastomosis of the gastric remnant, the site prone to carcinogenesis [8, 9]. Bile reflux may provide an explanation for the observed low prevalence of $H$. pylori in the stomach epithelium bordering stump carcinomas. Bile salts seem to have a bactericidal effect on $\mathrm{H}$. pylori and after a Billroth resection, H. pylori disappears rapidly from the gastroenterostoma.

In contrast, EBV infection appears more common in gastric stump cancer, not only in Asian countries, but in the western world as well. One possible explanation may be bile reflux into the stomach which could act as a cofactor mediating EBV infection of the epithelial cells, for instance by inducing fusion of EBV carrying B cells and epithelial cells. The EBV positive carcinomas were predominantly of the early type, which is consistent with previous observations that EBV infection perhaps occurs early in carcinogenesis [10].

A complete margin-negative (Ro) resection remains the only potentially curative treatment for gastric adenocarcinoma [11, 12]. Gastric stump carcinomas often have low resectability rates and a poor prognosis. Gastric stump carcinoma has a wide range of lymph node (LN) metastases, including $\mathrm{LN}$ within jejunal mesentery in Billroth II reconstruction cases. Therefore, it is suggested that radical operation for Billroth I patients needs removal of gastroduodenectomy anastomosis and the above LNs, and that Billroth II patients need removal of $10 \mathrm{~cm}$ of jejunum besides gastrojejunostomy anastomosis, and clearance of $\mathrm{LN}$ within its mesentery, in addition to Billroth I stump carcinoma $[13,14]$.

A recent study by Newman et al. supported the opinion that the outcome after resection in gastric stump carcinoma does not differ from that of patients with other primary proximal gastric cancers of the same stage. There are no differences in prognostic factors between primary gastric cancer and gastric stump cancer patients in terms of resectability rate, death rate, and survival. Therefore, the consequences of surgical therapy in these patients are identical [15].

\section{CONCLUSION}

Recommendations has been made to screen patients with gastro-jejunostomy by endoscopy at least annually to detect the cancer in early stages. Only a complete margin-negative resection offers chances of cure and mortality is very high in advanced cases of carcinoma stomach.

$$
* * * * * * * * *
$$

\section{Author Contributions}

Saroj Kant Sahoo - Conception and design, Analysis and interpretation of data, Critical revision of the article, Final approval of the version to be published

Manash Ranjan Sahoo - Acquisition of data, Analysis and interpretation of data, Critical revision of the article, Final approval of the version to be published

Ved Bhaskar - Acquisition of data, Drafting the article, Critical revision of the article, Final approval of the version to be published

\section{Guarantor}

The corresponding author is the guarantor of submission.

\section{Conflict of Interest}

Authors declare no conflict of interest.

\section{Copyright}

(C) Saroj Kant Sahoo et al. 2013; This article is distributed under the terms of Creative Commons Attribution 3.0 License which permits unrestricted use, distribution and reproduction in any means provided the original authors and original publisher are properly credited. (Please see www.ijcasereportsandimages.com /copyright-policy.php for more information.)

\section{REFERENCES}

1. Tanigawa N, Nomura E, Lee SW, et al. Current State of Gastric Stump Carcinoma in Japan: Based on the Results of a Nationwide Survey. World J Surg 2010;34(7):1540-7.

2. Taksdal S, Stalsberg H. Histology of gastric carcinoma occurring after gastric surgery for benign conditions. Cancer 1973;32(1):162-6. 
3. Littler ER, Gleibermann E. Gastritis cystica polyposa. (Gastric mucosal prolapse at gastroenterostomy site, with cystic and infiltrative epithelial hyperplasia). Cancer 1972;29(1):205-9.

4. Ken Kondo. Duodenogastric reflux and gastric stump carcinoma. Gastric Cancer 2002;5(1):16-22.

5. Qizilbash AH. Gastritis cystica and carcinoma arising in old gastrojejunostomy stoma. Can Med Assoc J 1975;112(12):1432-3.

6. Aoyagi K, Koufuji K, Yano S, et al. Two cases of cancer in the remnant stomach derived from gastritis cystica polyposa. Kurume Med J 2000;47(3):243-8.

7. Tanahashi Y, Ohwada S, Takubo K, et al. [A case of early carcinoma of the remnant stomach that developed from gastritis cystica polyposa]. Gan No Rinsho 1990;36(8):929-33.

8. Inge O Baas, Bastiaan P van Rees, Alex Musler, et al. Helicobacter pylori and Epstein-Barr virus infection and the p53 tumour suppressor pathway in gastric stump cancer compared with carcinoma in the nonoperated stomach. J Clin Pathol 1998;51(9):662-6.

9. Offerhaus GJ, Rieu PN, Jansen JB, Joosten HJ, Lamers CB. Prospective comparative study of the influence of bile reflux on human mucosal histology and Campylobacter pylori infection. Gut 1989;30(11):1552-7.
10. Inge $\mathrm{O}$ Baas, Bastiaan $\mathrm{P}$ van Rees, Alex Musler, et al Helicobacter pylori and Epstein-Barr virus infection and the p53 tumour suppressor pathway in gastric stump cancer compared with carcinoma in the nonoperated stomach. J Clin Pathol 1998;51(9):662-6.

11. Dicken BJ, Bigam DL, Cass C, Mackey JR, Joy AA, Hamilton SM. Gastric adenocarcinoma: Review and considerations for future directions. Ann Surg 2005;241(1):27-39.

12. Cho CS, Brennan MF: Gastric adenocarcinoma, in Cameron JL (ed): Current Surgical Therapy, gth ed. Philadelphia: Mosby, 2008.

13. Thorban S, Böttcher K, Etter M, Roder JD, Busch R, Siewert JR. Prognostic Factors in Gastric Stump Carcinoma. Ann Surg 2000;231(2):188-94.

14. Han SL, Hua YW, Wang CH, Ji SQ, Zhuang J. Metastatic pattern of lymph node and surgery for gastric stump cancer. J Surg Oncol 2003;82(4):241-6.

15. Mitsunobu Matsushita, Kiyoshi Hajiro, Kazuichi Okazaki, Hiroshi Takakuwa. Gastric Cancer Occurring at Anastomosis After Gastrojejunostomy Without gastrectomy. Dig Dis Sci 1998;43(4):898-900.
Access full text article on other devices

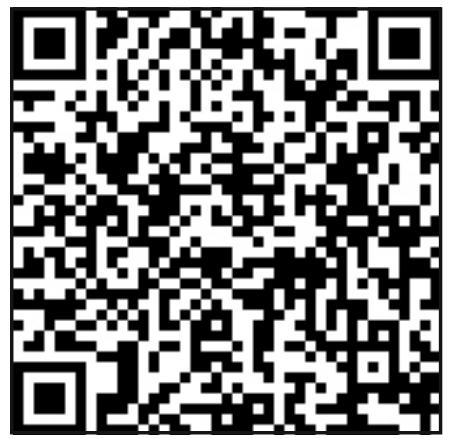

Access PDF of article on other devices

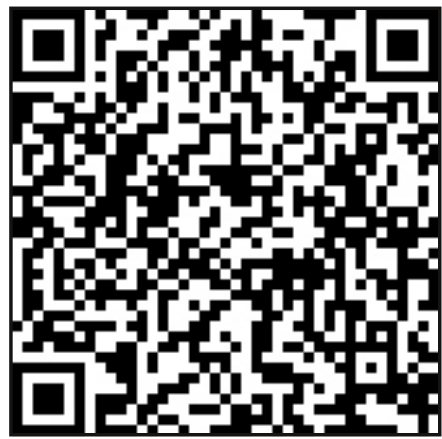

